\title{
Multiplex Amplifiable Probe Hybridization
}

National Cancer Institute

\section{Source}

National Cancer Institute. Multiplex Amplifiable Probe Hybridization. NCI Thesaurus. Code C116159.

A screening method used to determine abnormal DNA copy number changes in complex genomes. This method uses unique short probes which can be quantitatively recovered and amplified after hybridization to genomic DNA. In addition, the probes can be specifically designed for any genomic region of interest (such as locus, telomere, chromosomal segment, whole chromosome or total genome) with high hybridization specificity. 